\title{
Sign of Permutations Induced by Anick and Nagata-Anick Automorphisms over Finite Fields
}

\author{
Keisuke Hakuta \\ ${ }^{1}$ Interdisciplinary Graduate School of Science and Engineering, Shimane University, Shimane, Japan \\ Correspondence: Keisuke Hakuta, Interdisciplinary Graduate School of Science and Engineering, Shimane University, \\ 1060 Nishikawatsu-cho, Matsue, Shimane, Japan. Tel: 81-852-32-6518. E-mail: hakuta@cis.shimane-u.ac.jp
}

Received: March 23, 2017 Accepted: May 29, 2017 Online Published: July 3, 2017

doi:10.5539/jmr.v9n4p23 URL: https://doi.org/10.5539/jmr.v9n4p23

This work was supported by JSPS KAKENHI Grant-in-Aid for Young Scientists (B) 16 K16066.

\begin{abstract}
In this paper, we investigate the sign of permutations induced by the Anick automorphism and the Nagata-Anick automorphism over finite fields. We shall prove that if the Anick automorphism and the Nagata-Anick automorphism are defined over a prime field of characteristic two, they induce odd permutations, and otherwise, they induce even permutations.
\end{abstract}

Keywords: Polynomial automorphism, finite field, permutation, Anick automorphism, Nagata-Anick automorphism.

\section{Introduction}

Let $K$ be a field. We denote the polynomial ring in $n$ indeterminates $X_{1}, \ldots, X_{n}$ over $K$ by $K\left[X_{1}, \ldots, X_{n}\right]$. Let $K\left\langle X_{1}, \ldots, X_{n}\right\rangle$ denote the free associative algebra in $n$ indeterminates $X_{1}, \ldots, X_{n}$ over $K$. For polynomials $f_{1}, \ldots, f_{n} \in K\left[X_{1}, \ldots, X_{n}\right]$, the $n$-tuple of polynomials $F=\left(f_{1}, \ldots, f_{n}\right)$ is called a polynomial map. The set of polynomial maps over $K$ and the set of maps from $K^{n}$ to $K^{n}$ are denoted by $\operatorname{ME}_{n}(K)$ and $\operatorname{Maps}\left(K^{n}, K^{n}\right)$, respectively. Each polynomial map can be identified with a map from $K^{n}$ to $K^{n}$ via the following natural map

$$
\pi: \quad \operatorname{ME}_{n}(K) \rightarrow \operatorname{Maps}\left(K^{n}, K^{n}\right) .
$$

We denote by $\mathrm{GA}_{n}(K)$ (resp. $\mathrm{Aff}_{n}(K), \mathrm{EA}_{n}(K)$ ) the set of polynomial automorphisms (resp. affine automorphisms, elementary automorphisms) of $K^{n}$. We recall that

$$
\operatorname{Aff}_{n}(K) \cong K^{n} \rtimes G L_{n}(K)
$$

Let us denote by $\mathrm{TA}_{n}(K)$ the subgroup of $\mathrm{GA}_{n}(K)$ generated by two subgroups $\operatorname{Aff}_{n}(K)$ and $\mathrm{EA}_{n}(K)$. For $F \in \mathrm{GA}_{n}(K), F$ is called tame automorphism if $F \in \mathrm{TA}_{n}(K)$, and otherwise $\left(F \in \mathrm{GA}_{n}(K) \backslash \mathrm{TA}_{n}(K)\right) F$ is called wild automorphism. The Tame Generators Problem asks whether $\mathrm{GA}_{n}(K)=\mathrm{TA}_{n}(K)$, and is related to the Jacobian conjecture (See (Essen, 2000) for more details).

For any finite set $T$, we denote the symmetric group (resp. the alternating group) on $T$ by $\operatorname{Sym}(T)(\operatorname{resp}$. Alt $(T))$. Let sgn : $\operatorname{Sym}(T) \rightarrow\{ \pm 1\}$ be the sign function. If $K$ is a finite field $\mathbb{F}_{q}$ with $q$ elements $\left(p=\operatorname{char}\left(\mathbb{F}_{q}\right), q=p^{m}\right.$, and $\left.m \geq 1\right)$, we use the symbol $\pi_{q}$ instead of $\pi$ :

$$
\pi_{q}: \quad \operatorname{ME}_{n}\left(\mathbb{F}_{q}\right) \rightarrow \operatorname{Maps}\left(\mathbb{F}_{q}^{n}, \mathbb{F}_{q}^{n}\right) .
$$

When we restrict the map $\pi_{q}$ to $\mathrm{GA}_{n}\left(\mathbb{F}_{q}\right), \pi_{q}(G)$ is a subgroup of $\operatorname{Sym}\left(\mathbb{F}_{q}^{n}\right)$ for any subgroup $G \subseteq \mathrm{GA}_{n}\left(\mathbb{F}_{q}\right)$. Maubach has investigated the subgroup $\pi_{q}(G)$ in the case $G=\mathrm{TA}_{n}\left(\mathbb{F}_{q}\right)$ (Maubach, 2001).

Theorem 1. If $n \geq 2$, then $\pi_{q}\left(\mathrm{TA}_{n}\left(\mathbb{F}_{q}\right)\right)=\operatorname{Sym}\left(\mathbb{F}_{q}^{n}\right)$ if $q$ is odd or $q=2$. If $q=2^{m}$ where $m \geq 2$ then $\pi_{q}\left(\mathrm{TA}_{n}\left(\mathbb{F}_{q}\right)\right)=\operatorname{Alt}\left(\mathbb{F}_{q}^{n}\right)$.

If there exists $F \in \mathrm{GA}_{n}\left(\mathbb{F}_{2^{m}}\right)$ such that $\operatorname{sgn}\left(\pi_{2^{m}}(F)\right)=-1$, then we must have $F \in \mathrm{GA}_{n}\left(\mathbb{F}_{2^{m}}\right) \backslash \mathrm{TA}_{n}\left(\mathbb{F}_{2^{m}}\right)$. This indicates that the polynomial automorphism $F$ is wild. Thus, the following question is very important (Maubach, 2008).

Question 1. For $q=2^{m}$ and $m \geq 2$, do there exist polynomial automorphisms such that the permutations induced by the polynomial automorphisms belong to $\operatorname{Sym}\left(\mathbb{F}_{q}^{n}\right) \backslash \operatorname{Alt}\left(\mathbb{F}_{q}^{n}\right)$ ?

It is natural to consider the sign of the famous polynomial automorphisms such as the Nagata automorphism (Nagata, 1972), the Anick automorphism (Cohn, 2006), and the Nagata-Anick automorphism (Cohn, 2006). 
In this paper, we investigate the sign of permutations induced by the Anick automorphism and the Nagata-Anick automorphism over finite fields. We shall prove that if the Anick automorphism and the Nagata-Anick automorphism are defined over a prime field of characteristic two, they induce odd permutations, and otherwise, they induce even permutations.

\section{Sign of Permutations Induced by Anick Automorphism}

The Anick automorphism is defined by

$$
\delta:=(x+y(x y-y z), y, z+(x y-y z) y) \in \text { Aut }_{K} K\langle x, y, z\rangle .
$$

Remark that there exists a group homomorphism

$$
\theta: \operatorname{Aut}_{K} K\left\langle X_{1}, \ldots, X_{n}\right\rangle \rightarrow \mathrm{GA}_{n}(K)
$$

(See (Cohn, 2006) for details). We call $\pi_{q}(\theta(\delta))$ the permutation induced by the Anick automorphism. This section investigates the sign of the permutation induced by the Anick automorphism over a finite field. We begin with the following lemma.

Lemma 1. Let $\phi$, $\tau$ be elementary automorphisms defined by

$$
\phi:=(x, y, z-x), \quad \tau:=\left(x-y^{2} z, y, z\right) \in \mathrm{EA}_{3}(K) .
$$

Then we have

$$
\theta(\delta)=\phi^{-1} \circ \tau \circ \phi
$$

Proof. Since

$$
\begin{aligned}
\tau \circ \phi & =\left(x-y^{2} z, y, z\right) \circ(x, y, z-x) \\
& =\left(x-y^{2}(z-x), y, z-x\right) \\
& =(x+y(x y-y z), y, z-x),
\end{aligned}
$$

and $\phi^{-1}=(x, y, z+x)$, we have

$$
\begin{aligned}
\phi^{-1} \circ \tau \circ \phi & =(x+y(x y-y z), y, z-x+x+y(x y-y z)) \\
& =(x+y(x y-y z), y, z+y(x y-y z)) .
\end{aligned}
$$

Thus, $\theta(\delta)=\phi^{-1} \circ \tau \circ \phi$.

Next we prove Lemma 2 which is used to prove Main Theorem 1. Lemma 2 shows that the sign of $\pi_{q}(\tau)$ can be determined by Equation (4), where $\tau$ is the elementary automorphism defined in Lemma 1.

Lemma 2. (Sign of $\left.\pi_{q}(\tau)\right)$ Let $\tau$ be as in Lemma 1. Then we have

$$
\operatorname{sgn}\left(\pi_{q}(\tau)\right)=(-1)^{p^{m-1}(p-1)(q-1)^{2}} .
$$

Proof. Let $y_{0}, z_{0}$ be elements of $\mathbb{F}_{q}^{*}$. We define the map $\tau_{\left(y_{0}, z_{0}\right)}: \mathbb{F}_{q}^{3} \rightarrow \mathbb{F}_{q}^{3}$ as follows:

$$
\begin{aligned}
& \begin{array}{cccc}
\tau_{\left(y_{0}, z_{0}\right)}: & \underset{\mathbb{F}}{\mathbb{F}_{q}^{3}} \\
\Psi & & \begin{array}{c}
\mathbb{F}_{q}^{3} \\
\psi
\end{array}
\end{array} \\
& (x, y, z) \longmapsto\left(x-y^{2} z, y, z\right), \quad \text { if } y=y_{0} \text { and } z=z_{0}, \\
& (x, y, z) \longmapsto \quad(x, y, z), \quad \text { otherwise. }
\end{aligned}
$$

The map $\tau_{\left(y_{0}, z_{0}\right)}$ is obviously bijective. We set

$$
B\left(\tau_{\left(y_{0}, z_{0}\right)}\right):=\left\{(x, y, z) \in \mathbb{F}_{q}^{3} \mid \tau_{\left(y_{0}, z_{0}\right)}(x, y, z) \neq(x, y, z)\right\} .
$$

Since $B\left(\tau_{\left(y_{0}, z_{0}\right)}\right) \cap B\left(\tau_{\left(y_{0}^{\prime}, z_{0}^{\prime}\right)}\right)=\emptyset$ for any $\left(y_{0}^{\prime}, z_{0}^{\prime}\right) \in \mathbb{F}_{q}^{*} \times \mathbb{F}_{q}^{*} \backslash\left\{\left(y_{0}, z_{0}\right)\right\}$, it follows that

$$
\tau=\prod_{y_{0}, z_{0} \in \mathbb{F}_{q}^{*}} \tau_{\left(y_{0}, z_{0}\right)}
$$


which is a composition of disjoint permutations on $\mathbb{F}_{q}^{3}$. For $y_{0}, z_{0} \in \mathbb{F}_{q}^{*}$, we decompose each permutation $\tau_{\left(y_{0}, z_{0}\right)}$ as a composition of disjoint cycles on $\mathbb{F}_{q}^{3}$. In order to find such a decomposition, we define an equivalence relation $\stackrel{(\tau)}{\sim}$ on $\mathbb{F}_{q}$ : $x \in \mathbb{F}_{q}$ and $x^{\prime} \in \mathbb{F}_{q}$ are equivalent if and only if there exists $l \in\{0,1, \ldots, p-1\}$ such that $x^{\prime}=x-l y_{0}^{2} z_{0}$. Put

$$
C_{x}^{(\tau)}:=\left\{x^{\prime} \in \mathbb{F}_{q} \mid x \stackrel{(\tau)}{\sim} x^{\prime}\right\}
$$

We choose a complete system of representatives $\mathcal{R}_{\tau}$ for the above equivalence relation. Remark that $\sharp \mathcal{R}_{\tau}=q / p=p^{m} / p=$ $p^{m-1}$. For any $x_{0} \in \mathcal{R}_{\tau}$, we define the bijective map $\tau_{x_{0},\left(y_{0}, z_{0}\right)}: \mathbb{F}_{q}^{3} \rightarrow \mathbb{F}_{q}^{3}$ by

$$
\begin{aligned}
& \begin{array}{cccc}
\tau_{x_{0},\left(y_{0}, z_{0}\right)}: & \mathbb{F}_{q}^{3} & \longrightarrow & \mathbb{F}_{q}^{3} \\
\psi & & \psi^{2}
\end{array} \\
& (x, y, z) \longmapsto\left(x-y^{2} z, y, z\right), \quad \text { if } x \in C_{x_{0}}^{(\tau)}, y=y_{0}, \text { and } z=z_{0}, \\
& (x, y, z) \longmapsto \quad(x, y, z), \quad \text { otherwise. }
\end{aligned}
$$

It is easy to see that $\tau_{x_{0},\left(y_{0}, z_{0}\right)}$ is a cycle of length $p$, namely,

$$
\operatorname{sgn}\left(\pi_{q}\left(\tau_{x_{0},\left(y_{0}, z_{0}\right)}\right)\right)=(-1)^{p-1}
$$

Let $x_{0}^{\prime}$ be an element of $\mathcal{R}_{\tau}$. If $x_{0}^{\prime} \notin C_{x_{0}}^{(\tau)}$ then from $C_{x_{0}}^{(\tau)} \cap C_{x_{0}^{\prime}}^{(\tau)}=\emptyset$, we have

$$
\tau_{\left(y_{0}, z_{0}\right)}=\prod_{x_{0} \in \mathcal{R}_{\tau}} \tau_{x_{0},\left(y_{0}, z_{0}\right)},
$$

which is a composition of disjoint cycles on $\mathbb{F}_{q}^{3}$. Since $\pi_{q}$ and sgn are group homomorphisms, we obtain

$$
\begin{aligned}
\operatorname{sgn}\left(\pi_{q}(\tau)\right) & =\prod_{y_{0}, z_{0} \in \mathbb{F}_{q}^{*}, x_{0} \in \mathcal{R}_{\tau}} \operatorname{sgn}\left(\pi_{q}\left(\tau_{x_{0},\left(y_{0}, z_{0}\right)}\right)\right) \\
& =\prod_{y_{0}, z_{0} \in \mathbb{F}_{q}^{*}, x_{0} \in \mathcal{R}_{\tau}}(-1)^{p-1}=(-1)^{p^{m-1}(p-1)(q-1)^{2}} .
\end{aligned}
$$

This completes the proof of Lemma 2.

By Lemma 1 and Lemma 2, we obtain Main Theorem 1.

Main Theorem 1. (Sign of Anick automorphism) If $q$ is odd or $q=2^{m}, m \geq 2$ then we have $\pi_{q}(\theta(\delta)) \in \operatorname{Alt}\left(\mathbb{F}_{q}^{3}\right)$. If $q=2$ then we have $\pi_{q}(\theta(\delta)) \in \operatorname{Sym}\left(\mathbb{F}_{q}^{3}\right) \backslash \operatorname{Alt}\left(\mathbb{F}_{q}^{3}\right)$. Namely,

$$
\operatorname{sgn}\left(\pi_{q}(\theta(\delta))\right)= \begin{cases}1 & \left(q \text { is odd } \text { or } q=2^{m} \text { and } m \geq 2\right), \\ -1 & (q=2) .\end{cases}
$$

Proof. By the fact that $\pi_{q}$ and sgn are group homomorphisms and by Lemma 1, we have

$$
\begin{aligned}
\operatorname{sgn}\left(\pi_{q}(\theta(\delta))\right) & =\operatorname{sgn}\left(\pi_{q}\left(\theta\left(\phi^{-1} \circ \tau \circ \phi\right)\right)\right) \\
& =\operatorname{sgn}\left(\pi_{q}\left(\phi^{-1}\right) \pi_{q}(\tau) \pi_{q}(\phi)\right) \\
& =\operatorname{sgn}\left(\pi_{q}\left(\phi^{-1}\right)\right) \operatorname{sgn}\left(\pi_{q}(\tau)\right) \operatorname{sgn}\left(\pi_{q}(\phi)\right) \\
& =\operatorname{sgn}\left(\pi_{q}(\phi)\right)^{-1} \operatorname{sgn}\left(\pi_{q}(\tau)\right) \operatorname{sgn}\left(\pi_{q}(\phi)\right) \\
& =\operatorname{sgn}\left(\pi_{q}(\tau)\right) .
\end{aligned}
$$

Thus, it follows immediately from Lemma 2.

In (Drenski \& Yu, 2007), the following automorphism

$$
\omega:=(x+y(x y-y z), y, z+(x y-y z) y) \in \operatorname{Aut}_{K} K\langle x, y, z\rangle
$$

is called the Anick automorphism. These two Anick automorphisms $\theta(\delta)$ and $\theta(\omega)$ have the following relation:

$$
\theta(\omega)=\psi \circ \theta(\delta) \circ \psi,
$$

where $\psi=(x, z, y) \in \operatorname{Aff}_{3}(K)$. By Main Theorem 1 and by Equation (7), we obtain the following corollary (Corollary 1). 
Corollary 1. (Sign of Anick automorphism $\theta(\omega)$ ) Let $\delta \in \operatorname{Aut}_{\mathbb{F}_{q}} \mathbb{F}_{q}\langle x, y, z\rangle$ is the Anick automorphism defined by Equation (1), and let $\omega \in \operatorname{Aut}_{\mathbb{F}_{q}} \mathbb{F}_{q}\langle x, y, z\rangle$ is the Anick automorphism defined by Equation (6). Then we have

$$
\operatorname{sgn}\left(\pi_{q}(\theta(\delta))\right)=\operatorname{sgn}\left(\pi_{q}(\theta(\omega))\right) .
$$

Namely, the sign of the permutation induced by the Anick automorphism $\theta(\delta)$ is equal to that of the permutation induced by the Anick automorphism $\theta(\omega)$.

\section{Sign of Permutations Induced by Nagata-Anick Automorphism}

The Nagata-Anick automorphism is defined by

$$
\rho:=(w, x+(w x-y z) z, y+w(w x-y z), z) \in \operatorname{Aut}_{K} K\langle w, x, y, z\rangle .
$$

We call $\pi_{q}(\theta(\rho))$ the permutation induced by the Nagata-Anick automorphism, where $\theta$ is a group homomorphism defined by (2). In this section we consider the sign of the permutation induced by the Nagata-Anick automorphism over a finite field.

Let $t$ be a new variable. We define the polynomial $f(t) \in \mathbb{F}_{q}[t]$ as follows:

$$
f(t):=\prod_{c \in \mathbb{F}_{q}^{*}}(t-c) \in \mathbb{F}_{q}[t] .
$$

Choose a generator $g$ of the multiplicative group $\mathbb{F}_{q}^{*}$. Then one can easily see that

$$
\begin{aligned}
f(\alpha) & = \begin{cases}0 & \left(\alpha \in \mathbb{F}_{q}^{*}\right), \\
(-1)^{q-1} \prod_{i=0}^{q-2} g^{i} & (\alpha=0),\end{cases} \\
& = \begin{cases}0 & \left(\alpha \in \mathbb{F}_{q}^{*}\right), \\
(-1)^{q-1} g^{(q-2)(q-1) / 2} & (\alpha=0) .\end{cases}
\end{aligned}
$$

We put $c_{0}:=f(0)=(-1)^{q-1} g^{(q-2)(q-1) / 2}$. Remark that $c_{0} \in \mathbb{F}_{q}^{*}$. Setting $h(t):=c_{0}^{-1} \times f(t) \in \mathbb{F}_{q}[t]$ yields that

$$
h(\alpha)= \begin{cases}0 & \left(\alpha \in \mathbb{F}_{q}^{*}\right), \\ 1 & (\alpha=0) .\end{cases}
$$

We prove the following lemma (Lemma 3) which states that the permutation induced by the Nagata-Anick automorphism is equal to the permutation induced by a composition of four elementary automorphisms.

Lemma 3. Let $\psi, \xi$, $\lambda$ be elementary automorphisms defined by

$$
\begin{gathered}
\psi:=\left(w, x, y-w x z^{q-2}, z\right) \in \mathrm{EA}_{4}\left(\mathbb{F}_{q}\right), \\
\xi:=\left(w, x-y z^{2}, y, z\right) \in \mathrm{EA}_{4}\left(\mathbb{F}_{q}\right),
\end{gathered}
$$

and

$$
\lambda:=\left(w, x, y+w^{2} x h(z), z\right) \in \mathrm{EA}_{4}\left(\mathbb{F}_{q}\right) .
$$

Then we have

$$
\pi_{q}(\theta(\rho))=\pi_{q}\left(\lambda \circ \psi^{-1} \circ \xi \circ \psi\right) .
$$

Proof. By easy calculation, one can easily verify that

$$
\begin{aligned}
\pi_{q}(\xi \circ \psi) & =\pi_{q}\left(\left(w, x-y z^{2}, y, z\right) \circ\left(w, x, y-w x z^{q-2}, z\right)\right) \\
& =\pi_{q}\left(\left(w, x-\left(y-w x z^{q-2}\right) z^{2}, y-w x z^{q-2}, z\right)\right) \\
& =\pi_{q}\left(\left(w, x+(w x-y z) z, y-w x z^{q-2}, z\right)\right) .
\end{aligned}
$$

Since $\psi^{-1}=\left(w, x, y+w x z^{q-2}, z\right) \in \mathrm{EA}_{4}\left(\mathbb{F}_{q}\right)$, we have

$$
\begin{aligned}
& \pi_{q}\left(\psi^{-1} \circ \xi \circ \psi\right) \\
= & \pi_{q}\left(\left(w, x, y+w x z^{q-2}, z\right) \circ\left(w, x+(w x-y z) z, y-w x z^{q-2}, z\right)\right) \\
= & \pi_{q}\left(\left(w, x+(w x-y z) z, y-w x z^{q-2}+w(x+(w x-y z) z) z^{q-2}, z\right)\right) \\
= & \pi_{q}\left(\left(w, x+(w x-y z) z, y+w(w x-y z) z^{q-1}, z\right)\right) .
\end{aligned}
$$


Therefore,

$$
\begin{aligned}
& \pi_{q}\left(\lambda \circ \psi^{-1} \circ \xi \circ \psi\right) \\
= & \pi_{q}\left(\left(w, x, y+w^{2} x h(z), z\right) \circ\left(w, x+(w x-y z) z, y+w(w x-y z) z^{q-1}, z\right)\right) \\
= & \pi_{q}\left(\left(w, x+(w x-y z) z, y+w(w x-y z) z^{q-1}+w^{2}(x+(w x-y z) z) h(z), z\right)\right) \\
= & \pi_{q}(\theta(\rho)) .
\end{aligned}
$$

Hence we obtain Equation (10).

Remark 1. In the case of the Anick automorphism, Equation (3) holds as polynomial automorphisms. On the other hand, in the case of the Nagata-Anick automorphism, we have

$$
\theta(\rho) \neq \lambda \circ \psi^{-1} \circ \xi \circ \psi .
$$

Remark that Equation (10) holds as permutations on $\mathbb{F}_{q}^{4}$.

We use the same argument as in Lemma 1 in order to show the main result of this section (Main Theorem 2). Namely, we consider the sign of $\pi_{q}(\lambda)$ and $\pi_{q}(\xi)$ which are defined in Lemma 3.

Lemma 4. Let $\lambda$ be as in Lemma 3. Then we have

$$
\operatorname{sgn}\left(\pi_{q}(\lambda)\right)=(-1)^{p^{m-1}(p-1)(q-1)^{2}} .
$$

Proof. Let $w_{0}, x_{0}$ be elements of $\mathbb{F}_{q}^{*}$. We define the map $\lambda_{\left(w_{0}, x_{0}\right)}: \mathbb{F}_{q}^{4} \rightarrow \mathbb{F}_{q}^{4}$ as follows:

$$
\begin{aligned}
& \begin{array}{ccc}
\lambda_{\left(w_{0}, x_{0}\right)}: & \mathbb{F}_{q}^{4} \\
\Psi & \longrightarrow & \mathbb{F}_{q}^{4} \\
\Psi
\end{array} \\
& (w, x, y, z) \longmapsto\left(w, x, y+w^{2} x h(z), z\right), \quad \text { if }(w, x, z)=\left(w_{0}, x_{0}, 0\right), \\
& (w, x, y, z) \longmapsto \quad(w, x, y, z), \quad \text { otherwise. }
\end{aligned}
$$

The map $\lambda_{\left(w_{0}, x_{0}\right)}$ is obviously bijective. We set

$$
B\left(\lambda_{\left(w_{0}, x_{0}\right)}\right):=\left\{(w, x, y, z) \in \mathbb{F}_{q}^{4} \mid \lambda_{\left(w_{0}, x_{0}\right)}(w, x, y, z) \neq(w, x, y, z)\right\} .
$$

Since $B\left(\lambda_{\left(w_{0}, x_{0}\right)}\right) \cap B\left(\lambda_{\left(w_{0}^{\prime}, x_{0}^{\prime}\right)}\right)=\emptyset$ for any $\left(w_{0}^{\prime}, x_{0}^{\prime}\right) \in \mathbb{F}_{q}^{*} \times \mathbb{F}_{q}^{*} \backslash\left\{\left(w_{0}, x_{0}\right)\right\}$, it follows that

$$
\lambda=\prod_{w_{0}, x_{0} \in \mathbb{F}_{q}^{*}} \lambda_{\left(w_{0}, x_{0}\right)},
$$

which is a composition of disjoint permutations on $\mathbb{F}_{q}^{4}$. For $w_{0}, x_{0} \in \mathbb{F}_{q}^{*}$, we decompose each permutation $\lambda_{\left(w_{0}, x_{0}\right)}$ as a composition of disjoint cycles on $\mathbb{F}_{q}^{4}$. In order to find such a decomposition, we define an equivalence relation $\stackrel{(\lambda)}{\sim}$ on $\mathbb{F}_{q}$ : $y \in \mathbb{F}_{q}$ and $y^{\prime} \in \mathbb{F}_{q}$ are equivalent if and only if there exists $l \in\{0,1, \ldots, p-1\}$ such that $y^{\prime}=y+l w_{0}^{2} x_{0}$. Put

$$
C_{y}^{(\lambda)}:=\left\{y^{\prime} \in \mathbb{F}_{q} \mid y \stackrel{(\lambda)}{\sim} y^{\prime}\right\} .
$$

We choose a complete system of representatives $\mathcal{R}_{\lambda}$ for the above equivalence relation. Remark that $\sharp \mathcal{R}_{\lambda}=q / p=p^{m} / p=$ $p^{m-1}$. For any $y_{0} \in \mathcal{R}_{\lambda}$, we define the bijective map $\lambda_{y_{0},\left(w_{0}, x_{0}\right)}: \mathbb{F}_{q}^{4} \rightarrow \mathbb{F}_{q}^{4}$ by

$$
\begin{aligned}
& \begin{array}{llll}
\lambda_{y_{0},\left(w_{0}, x_{0}\right)}: & \mathbb{F}_{q}^{4} & \longrightarrow & \mathbb{F}_{q}^{4} \\
\psi & & \psi^{4}
\end{array} \\
& (w, x, y, z) \longmapsto\left(w, x, y+w^{2} x h(z), z\right), \\
& \text { if }(w, x, z)=\left(w_{0}, x_{0}, 0\right), y \in C_{y_{0}}^{(\lambda)} \text {, and } z=0 \text {, } \\
& (w, x, y, z) \longmapsto \quad(w, x, y, z), \quad \text { otherwise. }
\end{aligned}
$$

It is easy to see that $\lambda_{y_{0},\left(w_{0}, x_{0}\right)}$ is a cycle of length $p$, namely,

$$
\operatorname{sgn}\left(\pi_{q}\left(\lambda_{y_{0},\left(w_{0}, x_{0}\right)}\right)\right)=(-1)^{p-1} .
$$


Let $y_{0}^{\prime}$ be an element of $\mathcal{R}_{\lambda}$. If $y_{0}^{\prime} \notin C_{y_{0}}^{(\lambda)}$ then from $C_{y_{0}}^{(\lambda)} \cap C_{y_{0}^{\prime}}^{(\lambda)}=\emptyset$, we have

$$
\lambda_{\left(w_{0}, x_{0}\right)}=\prod_{y_{0} \in \mathcal{R}_{\lambda}} \lambda_{y_{0},\left(w_{0}, x_{0}\right)},
$$

which is a composition of disjoint cycles on $\mathbb{F}_{q}^{4}$. Since $\pi_{q}$ and sgn are group homomorphisms, we obtain

$$
\begin{aligned}
\operatorname{sgn}\left(\pi_{q}(\lambda)\right) & =\prod_{w_{0}, x_{0} \in \mathbb{E}_{q}^{*}, y_{0} \in \mathcal{R}_{\lambda}} \operatorname{sgn}\left(\pi_{q}\left(\lambda_{y_{0},\left(w_{0}, x_{0}\right)}\right)\right) \\
& =\prod_{w_{0}, x_{0} \in \mathbb{F}_{q}^{*}, y_{0} \in \mathcal{R}_{\lambda}}(-1)^{p-1}=(-1)^{p^{m-1}(p-1)(q-1)^{2}} .
\end{aligned}
$$

This completes the proof of Lemma 4.

Lemma 5. Let $\xi$ be as in Lemma 3. Then we have

$$
\operatorname{sgn}\left(\pi_{q}(\xi)\right)=(-1)^{p^{m-1}(p-1)(q-1)^{2} q} .
$$

Proof. Let $y_{0}, z_{0}$ be elements of $\mathbb{F}_{q}^{*}$. We define the map $\xi_{\left(y_{0}, z_{0}\right)}: \mathbb{F}_{q}^{4} \rightarrow \mathbb{F}_{q}^{4}$ as follows:

$$
\begin{array}{rllll}
\xi_{\left(y_{0}, z_{0}\right)}: & \mathbb{F}_{q}^{4} & \longrightarrow & \mathbb{F}_{q}^{4} & \\
\Psi & & \Psi & \\
(w, x, y, z) & \longmapsto & \left(w, x-y z^{2}, y, z\right), & & \text { if }(y, z)=\left(y_{0}, z_{0}\right), \\
(w, x, y, z) & \longmapsto & (w, x, y, z), & & \text { otherwise. }
\end{array}
$$

The map $\xi_{\left(y_{0}, z_{0}\right)}$ is obviously bijective. We set

$$
B\left(\xi_{\left(y_{0}, z_{0}\right)}\right):=\left\{(w, x, y, z) \in \mathbb{F}_{q}^{4} \mid \xi_{\left(y_{0}, z_{0}\right)}(w, x, y, z) \neq(w, x, y, z)\right\} .
$$

Since $B\left(\xi_{\left(y_{0}, z_{0}\right)}\right) \cap B\left(\xi_{\left(y_{0}^{\prime}, z_{0}^{\prime}\right)}\right)=\emptyset$ for any $\left(y_{0}^{\prime}, z_{0}^{\prime}\right) \in \mathbb{F}_{q}^{*} \times \mathbb{F}_{q}^{*} \backslash\left\{\left(y_{0}, z_{0}\right)\right\}$, it follows that

$$
\xi=\prod_{y_{0}, z_{0} \in \mathbb{F}_{q}^{*}} \xi_{\left(y_{0}, z_{0}\right)}
$$

which is a composition of disjoint permutations on $\mathbb{F}_{q}^{4}$. For $y_{0}, z_{0} \in \mathbb{F}_{q}^{*}$, we decompose each permutation $\xi_{\left(y_{0}, z_{0}\right)}$ as a composition of disjoint cycles on $\mathbb{F}_{q}^{4}$. In order to find such a decomposition, we define an equivalence relation $\stackrel{(\xi)}{\sim}$ on $\mathbb{F}_{q}$ : $x \in \mathbb{F}_{q}$ and $x^{\prime} \in \mathbb{F}_{q}$ are equivalent if and only if there exists $l \in\{0,1, \ldots, p-1\}$ such that $x^{\prime}=x-l y_{0} z_{0}^{2}$. Put

$$
C_{x}^{(\xi)}:=\left\{x^{\prime} \in \mathbb{F}_{q} \mid x \stackrel{(\xi)}{\sim} x^{\prime}\right\}
$$

We choose a complete system of representatives $\mathcal{R}_{\xi}$ for the above equivalence relation. Remark that $\sharp \mathcal{R}_{\xi}=q / p=p^{m} / p=$ $p^{m-1}$. For any $x_{0} \in \mathcal{R}_{\xi}$, we define the bijective map $\xi_{\left(w_{0}, x_{0}\right),\left(y_{0}, z_{0}\right)}: \mathbb{F}_{q}^{4} \rightarrow \mathbb{F}_{q}^{4}$ by

$$
\begin{aligned}
& \begin{array}{cccc}
\xi_{\left(w_{0}, x_{0}\right),\left(y_{0}, z_{0}\right)}: & \mathbb{F}_{q}^{4} \\
\Psi & & & \mathbb{F}_{q}^{4} \\
\psi & & \Psi
\end{array} \\
& (w, x, y, z) \longmapsto\left(w, x-y z^{2}, y, z\right), \\
& (w, x, y, z) \quad \text { if }(w, y, z)=\left(w_{0}, y_{0}, z_{0}\right) \text { and } x \in C_{x_{0}}^{(\xi)} \text {, }
\end{aligned}
$$

It is easy to see that $\xi_{\left(w_{0}, x_{0}\right),\left(y_{0}, z_{0}\right)}$ is a cycle of length $p$, namely,

$$
\operatorname{sgn}\left(\pi_{q}\left(\xi_{\left(w_{0}, x_{0}\right),\left(y_{0}, z_{0}\right)}\right)\right)=(-1)^{p-1} .
$$

Let $x_{0}^{\prime}$ be an element of $\mathcal{R}_{\xi}$. If $x_{0}^{\prime} \notin C_{x_{0}}^{(\xi)}$ then from $C_{x_{0}}^{(\xi)} \cap C_{x_{0}^{\prime}}^{(\xi)}=\emptyset$, we have

$$
\xi_{\left(y_{0}, z_{0}\right)}=\prod_{w_{0} \in \mathbb{F}_{q}, x_{0} \in \mathcal{R}_{\xi}} \xi_{\left(w_{0}, x_{0}\right),\left(y_{0}, z_{0}\right)} .
$$


which is a composition of disjoint cycles on $\mathbb{F}_{q}^{4}$. Since $\pi_{q}$ and sgn are group homomorphisms, we obtain

$$
\begin{aligned}
\operatorname{sgn}\left(\pi_{q}(\xi)\right) & =\prod_{w_{0} \in \mathbb{F}_{q}, x_{0} \in \mathcal{R}_{\xi}, y_{0}, z_{0} \in \mathbb{F}_{q}^{*}} \operatorname{sgn}\left(\pi_{q}\left(\xi_{\left(w_{0}, x_{0}\right),\left(y_{0}, z_{0}\right)}\right)\right) \\
& =\prod_{w_{0} \in \mathbb{F}_{q}, x_{0} \in \mathcal{R}_{\xi}, y_{0}, z_{0} \in \mathbb{F}_{q}^{*}}(-1)^{p-1}=(-1)^{p^{m-1}(p-1)(q-1)^{2} q} .
\end{aligned}
$$

This completes the proof of Lemma 5.

By Lemma 3 through Lemma 5, we obtain Main Theorem 2.

Main Theorem 2. (Sign of Nagata-Anick automorphism) If $q$ is odd or $q=2^{m}, m \geq 2$ then we have $\pi_{q}(\theta(\rho)) \in \operatorname{Alt}\left(\mathbb{F}_{q}^{4}\right)$. If $q=2$ then we have $\pi_{q}(\theta(\rho)) \in \operatorname{Sym}\left(\mathbb{F}_{q}^{4}\right) \backslash \operatorname{Alt}\left(\mathbb{F}_{q}^{4}\right)$. Namely,

$$
\operatorname{sgn}\left(\pi_{q}(\theta(\rho))\right)= \begin{cases}1 & \left(q \text { is odd or } q=2^{m} \text { and } m \geq 2\right), \\ -1 & (q=2) .\end{cases}
$$

Proof. By the fact that $\pi_{q}$ and sgn are group homomorphisms and by Lemma 3, we have

$$
\begin{aligned}
\operatorname{sgn}\left(\pi_{q}(\theta(\rho))\right) & =\operatorname{sgn}\left(\pi_{q}\left(\theta\left(\lambda \circ \psi^{-1} \circ \xi \circ \psi\right)\right)\right) \\
& =\operatorname{sgn}\left(\pi_{q}(\lambda) \pi_{q}\left(\psi^{-1}\right) \pi_{q}(\xi) \pi_{q}(\psi)\right) \\
& =\operatorname{sgn}\left(\pi_{q}(\lambda)\right) \operatorname{sgn}\left(\pi_{q}\left(\psi^{-1}\right)\right) \operatorname{sgn}\left(\pi_{q}(\xi)\right) \operatorname{sgn}\left(\pi_{q}(\psi)\right) \\
& =\operatorname{sgn}\left(\pi_{q}(\lambda)\right) \operatorname{sgn}\left(\pi_{q}(\psi)\right)^{-1} \operatorname{sgn}\left(\pi_{q}(\xi)\right) \operatorname{sgn}\left(\pi_{q}(\psi)\right) \\
& =\operatorname{sgn}\left(\pi_{q}(\lambda)\right) \operatorname{sgn}\left(\pi_{q}(\xi)\right) .
\end{aligned}
$$

Thus, it follows immediately from Lemma 4 and Lemma 5.

Remark 2. We derive the sign of permutation induced by the Anick automorphism (Main Theorem 1) by using Lemma 1 and Lemma 2, and also derive the sign of permutation induced by the Nagata-Anick automorphism (Main Theorem 2) by using Lemma 3, Lemma 4, and Lemma 5. Our strategy might be used in the Nagata automorphism case if one can prove the similar results to Lemma 1 and Lemma 2 for the Anick automorphism case, and Lemma 3 through Lemma 5 for the Nagata-Anick automorphism case.

\section{References}

Cohn, P. M. (2006). Free Ideal Rings and Localization in General Rings. Cambridge University Press. https://doi.org/10.1017/CBO9780511542794

Drenski, V., \& Yu, J.-T. (2007). The strong Anick conjecture is true. J. European Math. Soc., 9(4), 659-679. http://doi.org/10.4171/JEMS/92

van den Essen, A. (2000). Polynomial Automorphisms and the Jacobian Conjecture. Birkhäuser. https://doi.org/10.1007/978-3-0348-8440-2

Maubach, S. (2001). Polynomial automorphisms over finite fields. Serdica Math. J., 27(4), 343-350.

Maubach, S. (2008). A problem on polynomial maps over finite fields. arXiv preprint, arXiv:0802.0630.

Nagata, M. (1972). On automorphism group of $k[x, y]$. Kinokuniya Book Store Co. Ltd.

\section{Copyrights}

Copyright for this article is retained by the author(s), with first publication rights granted to the journal.

This is an open-access article distributed under the terms and conditions of the Creative Commons Attribution license (http://creativecommons.org/licenses/by/4.0/). 